\title{
La réforme de la fonction publique en Afrique subsaharienne: cas de la République Démocratique du Congo
}

\author{
Richard Tshienda Muambi \\ Faculté de Droit, Université de Kinshasa, République Démocratique du Congo
}

\begin{abstract}
Many reforms of the public service took place during the last thirty years on the African continent. Different strategies have been implemented to modernize and improve the functioning of administrative structures. The objective pursued by this paper is to understand the strategies of reform of the public service applied by the Democratic Republic of Congo and to try to evaluate the results.

Key words: reform of the public service, institutional tension, management tension and legitimation tension
\end{abstract}

\begin{abstract}
De nombreuses réformes de la fonction publique ont eu lieu au cours des trente dernières années dans le continent africain. Des stratégies différentes ont été mises en œuvre afin de moderniser et d'améliorer le fonctionnement des structures administratives. L'objectif que poursuit cet article est de comprendre les stratégies déployées dans la conduite de réforme de la fonction publique en République Démocratique du Congo et d'essayer d'en évaluer les résultats.

Mots clés: réforme de la fonction publique, tension institutionnelle, tension managériale et tension légitimationnelle
\end{abstract}

\section{Introduction}

Cet article vise d'une part à identifier les facteurs et les enjeux de la réforme de la fonction publique entreprise depuis trois décennies en Afrique subsaharienne en général et d'autre part, à appréhender les différentes stratégies déployées par la République Démocratique du Congo dans la réforme de sa fonction publique ainsi qu'en évaluer les résultats.

Sur la base d'une étude de type qualitatif, mêlant entretiens et approche socio-historique, nous procédons d'abord à la description des stratégies de réforme administrative appliquées en République Démocratique du Congo et ensuite, à l'évaluation de leur efficacité, voire de leur pertinence quant à l'aboutissement de ce processus de mutations.

Grâce aux entrevues que nous avons réalisées avec certaines parties prenantes à ce processus, à sept années d'observations en qualité d'expert du Groupe Projet Fonction Publique (GPFP), et enfin à la revue de la littérature se rapportant à notre sujet que nous avons pu examiner les changements projetés. 
Pour mener à bien la présente étude, il convient d'abord de situer le cadre théorique et les hypothèses qui orientent notre réflexion. Ensuite, nous allons mettre en lumière la méthodologie employée. Et enfin, nous essaierons de révéler les tendances, somme toute, générales des réformes de la fonction publique en Afrique subsaharienne en général et en République Démocratique du Congo en particulier.

\section{Fondements théoriques de la recherche}

Le cadre théorique de notre étude s'inscrit dans le champ de la sociologie des organisations, de la science administrative et du droit public. Les réflexions des sociologues, des politologues ainsi que des juristes publicistes nous permettront de répondre aux questions qui nous préoccupent: les facteurs et enjeux de la réforme de la fonction publique en Afrique subsaharienne; le contenu de cette réforme en République Démocratique du Congo; son bilan et les perspectives qu'elle augure.

En premier lieu, nous allons procéder à l'analyse des tensions qui sous-tendent toute réforme administrative. En effet celle-ci oscille entre le pôle économique et le pôle social des changements à opérer ${ }^{\mathrm{I}}$.

En règle générale, les organisations doivent réaliser des missions économiques (productivité du personnel, projets, missions à accomplir, prestations à fournir), de même qu'elles doivent prendre en compte des missions sociales (satisfaction et motivation du personnel, prise en compte des projets professionnel et personnel, des objectifs de carrière des employés) ${ }^{2}$.

Pour sa part, Yves Frédéric Livian soutient que l'analyse des organisations a toujours correspondu au triptyque: stratégie-structure-culture (Livian, I998, 226)3. En effet, toute réforme organisationnelle, notamment celle menée dans le secteur public, est confrontée à la gestion de tensions en vue d'assurer un équilibre institutionnel permettant, ainsi, la pérennité du fonctionnement des unités administratives ${ }^{4}$.

Les trois éléments qui constituent le triptyque susmentionné correspondent, dans l'étude des organisations, à la tension institutionnelle (stratégie), la tension managériale ou de gestion (structure) et la tension légitimationnelle (culture) 5 .

La tension institutionnelle permet l'étude des organisations dans leur contexte institutionnel propre et singulier. L'État et les lois qu'il adopte, les aspirations ou attentes des citoyens, des usagers, des politiques par rapport aux organisations sont autant de paramètres dont il faut tenir compte dans l'analyse de toute organisation. Cette dimension est d'autant plus importante, car elle est la première tension à laquelle l'organisation doit

\footnotetext{
I D. Giauque et D. J. Caron, op. cit., "Réformes administratives et gestions des ressources humaines: comparaison de la Suisse et du Canada ", De Boeck Supérieur, Revue internationale de politique comparée, 2004, 2004/2, Vol. II, p. 226.

2 D. Giauque et D. J. Caron, op. cit., p. 226.

3 Y. F. Livian cité par D.Giauque et D. J. Caron, «Réformes administratives et gestions des ressources humaines: comparaison de la Suisse et du Canada ", De Boeck Supérieur, Revue internationale de politique comparée, 2004, 2004/2, Vol. II, p.226.

D. Giauque et D. J. Caron, op. cit., p. 226.

5

F. Piotet et R. Sainsaulieu, Méthodes pour une sociologie de l'entreprise, Paris, Presses de la Fondation nationale des sciences politiques, I994, 374 .
} 
faire face. De ce fait, les organisations doivent braver une double contrainte : d'une part les contraintes provenant de leur environnement immédiat et d'autre part les contraintes internes liées à la production.

La deuxième tension, quant à elle, est celle liée à la gestion, c'est-à-dire les relations de pouvoir, les canaux de circulation des informations entre différents sphères de l'organisation, la gestion proprement dite (ses principes, ses procédures, ses règles, etc.), tous ces éléments sont à prendre en compte et font partie de cette dimension managériale des organisations.

Enfin, la troisième tension touche à la légitimation des objectifs que s'est fixés l'organisation et des pratiques qui y prévalent. Pour atteindre le but poursuivi par n'importe quelle organisation, il faudrait que tous les acteurs puissent regarder dans la même direction. "Ceci suppose en effet que l'organisation ait légitimé les règles et les pratiques en vigueur. Tout le défi est ici d'aboutir à un consensus organisationnel pour réaliser une justification durable des différents objectifs, règles et normes des organisations pour intégrer 'subjectivement' les acteurs en vue de la réalisation d'objectifs qui doivent être légitimés ${ }^{6}$."

En second lieu, avant d'avancer nos hypothèses, nous tenons à préciser notre conception de la réforme administrative et de celle de la fonction publique, car estimonsnous, que ces deux notions sont liées et la première est plus englobante que la deuxième et peut avoir des implications sur celle-ci.

En effet, la réforme administrative évoque l'idée de changement. Il ne s'agit bien sûr pas de n'importe quel changement, mais dans le cas d'une réforme administrative, celui-ci doit être planifié7.

«Action volontaire et concertée, la réforme administrative n'est jamais le fait du hasard, mais le résultat d'un processus dont on peut déceler les origines et les diverses étapes jusqu'au moment où il est fait un sort à la réforme ${ }^{8}$."

«À l'encontre des cas bien plus nombreux et probablement plus importants où l'administration se contente de s'adapter aux changements survenus dans son environnement, il s'agit de cas de génie social ou de "design» organisationnel, où un ou des réformateurs essaient délibérément de changer le cours de l'évolution institutionnelle9.» Pareille définition implique, selon Michel Crozier et Vincent Lemieux, l'identifica-

E. Reynaud et J.-D Reynaud, «La régulation conjointe et ses dérèglements », Le Travail humain, vol. 57, n I, I994, pp. 227-238.

7 G. Caiden, "Administrative reform", RISA, I968, pp.347 et s. repris dans Y.Chapel, "Stratégies et tactiques de la modernisation publique dans les pays en développement» (recueil de textes), Bruxelles, IISA, I972, p.I8. Voir aussi du même auteur, «Administrative reform : a Prospectus ", RISA), vol. 44, nos I et 2, I978, pp. Io6 à I20 et et Kenneth Cabatoff, "La réforme institutionnelle de la fonction publique», dans J. Bauer et K. Cabatoff, «Bilan de la loi 50: dynamique du changement de la fonction publique québécoise, Actes du premier colloque sur la réforme et le changement en administration publique ", Universitédu Québec à Montréal, les 20-2I mars I980, pp. 37 à 59.

8 J. I. Gow, «La réforme institutionnelle de la fonction publique de I983 : contexte, contenu et enjeux", Politique, $\mathrm{n}^{\circ} 6, \mathrm{r} 984, \mathrm{p} .54$.

9 M. Crozier et E. Friedberg, L'acteur et le système, Paris, Le Seuil, I977, pp. 2 I2 à 2I4. Voir également V. Lemieux, Les cheminements de l'influence, Québec, PUL, I979, p. 5. 
tion des participants à un processus de réforme ainsi que des valeurs privilégiées par ces acteurs. Activité consciente et finalisée, la réforme administrative se prête bien au genre $\mathrm{d}^{\prime}$ analyse stratégique ${ }^{\mathrm{I0}}$.

A ce stade, il sied de souligner qu'une bonne partie de la science administrative se préoccupe de problème de la réforme administrative, depuis l'organisation scientifique du travail jusqu'aux méthodes les plus récentes de prise de décision et d'analyse budgétaire.

Selon l'angle d'attaque que l'on adopte, le processus de réforme visé mettra l'accent soit sur des facteurs internes, soit sur les facteurs externes de l'organisation. "La tendance de la science administrative normative est de privilégier les facteurs internes; par exemple BUTANI écrit qu'une réforme administrative ne pourra réussir que si l'urgence de la réforme est comprise par l'organisation, si ceux qui devront l'appliquer sont associés à sa conception, que si un organe ad hoc est créé pour assurer le suivi et l'évaluation de la réforme et que si le personnel qui dirigera la réforme est choisi et formé soigneusement $^{\mathrm{II}}$.»

«Ce genre d'idée inspire l'école du développement organisationnel selon laquelle il faut dégeler les attitudes des membres d'une organisation afin de les rendre réceptifs à la réforme ${ }^{\mathrm{I} 2}$ ".

En revanche, l'approche des sociologues préoccupés par l'adéquation des structures aux exigences de leur environnement oriente notre attention sur le leadership, le choix de la technologie appropriée à adopter et le maintien de liens avec les groupes dans les milieux desquels l'appui est essentiel pour obtenir les ressources nécessaires à la survie de l'institution ${ }^{13}$.

Il importe de noter que la littérature de science administrative nous suggère deux remarques. En effet, la réforme administrative s'inspire de valeurs économiques (l'efficience, l'efficacité et l'économie) ou bien politiques (l'égalité, la représentativité, l'impartialité et la liberté) ${ }^{\mathrm{I} 4}$.

La plupart des réformes tenteront de jouer sur les deux registres, c'est-à-dire, essaieront de promouvoir les deux types de valeur en même temps, mais on conçoit facilement qu'elles puissent être en contradiction les unes avec les autres. Finalement, la réforme administrative aura des conséquences attendues par ses auteurs, mais elle en aura d'autres qui ne seront ni attendues ni voulues ${ }^{15}$.

Par ailleurs, les réformes qu'elles soient ponctuelles ou globales se heurtent souvent à une série d'obstacles considérables, de cercles vicieux qui en rendent la réalisation délicate. C'est notamment les cas d'absence ou inadaptation de structures d'encadrement

M. Crozier et E. Friedberg, op. cit., p. 5 .

K. N. Butani, cité par J. Iain Gow, op. cit., p.55, «Implementing Administrative Innovations and Reforms", The Indian Journal of Public Administration, July-Sept. I966, reproduit dans Y. Chapel, op. cit. pp., 39 à 42.

I2 J. I. Gow, op. cit., p.56.

I3 M. Chevallier et D. Loschak, Science administrative, vol. I, Paris, LGDJ, I979, p. 238.

I4 K. Cabatoff, op. cit., p. 4I.

I5 Idem, op. cit., p. 4I. 
de la réforme "au sommet», manque de cadres, incompétence, absentéisme, manque de moyens financiers, patrimonialisme, etc. Au-delà du problème d'encadrement et de la qualité des travaux de réforme, revient sans cesse le problème de volonté politique qui est le préalable incontournable de succès.

En revanche, appréhender la fonction publique comme appareil institutionnel, c'est privilégier l'étude des interrelations qui l'unissent au reste de la société et chercher à comprendre la logique sociale qui sous-tend son action.

Suivant le raisonnement que nous empruntons à Vincent Lemieux, il y a donc possibilité d'intégrer ces différentes approches (juridique et sociologique, limitée ou extensive) de la notion de la fonction publique par le recours à la notion du politique ${ }^{\mathrm{i} 6}$.

En effet, Lemieux considère que «le politique consiste dans les relations de pouvoir par lesquelles se fait la gouvernance des organisations ». En tant que systèmes finalisés d'action, les organisations doivent assurer la régulation des rapports entre leurs composantes ainsi que ceux qui les engagent vis-à-vis des intervenants dans leur environnement $^{17}$.

C'est pour cette raison que dans le cadre de la présente étude, nous optons pour une définition extensive des termes fonction publique, définition qui comprend d'une part les règles de sa gouvernance et d'autre part la chose instituée, c'est-à-dire l'ensemble des personnes qui la composent à un moment donné.

C'est à la fonction publique entendue dans ses deux acceptions, fonctionnelle et organique que la présente contribution est consacrée.

$\mathrm{Vu}$ sous cet angle, la réforme de la fonction publique est, selon nous, un train de mesures destinées à permettre à l'administration d'accomplir ses missions en tenant compte des critères de coût, d'efficacité et de qualité.

En tout état de causes, il s'agit ici d'atténuer les rigidités internes et externes liées à la gestion publique par le passage à une structure organisationnelle plus ouverte et plus souple. La prise de conscience de cette exigence a conduit, en France comme dans les autres États membres de l'Organisation de coopération et développement économique, OCDE en sigle, à engager depuis les années I980 un processus de réforme ${ }^{\mathrm{I} 8}$.

Partant de la définition de ces trois tensions organisationnelles qui se dégagent de la sociologie d'une part et de la conception que nous nous faisons de la réforme de la fonction publique d'autre part, nous posons que la réforme de la fonction publique constitue, dans bien des cas, un préalable à toute activité de développement rationnelle et continue dans un Etat. L'insuccès qui caractérise la réforme de la fonction publique en République Démocratique du Congo a comme principale cause le manque de volonté politique de la part des autorités de ce pays.

Pour réussir, une réforme doit s'inspirer d'une volonté de changement, elle doit trouver un catalyseur pour l'initier et la poursuivre, elle doit obtenir la collaboration, dans le meilleur des mondes, de tous les fonctionnaires, sinon d'une bonne partie de ceux-ci et doit bénéficier du soutien des acteurs de différents milieux environnant ou bien apporter

I8 J. Chevallier, «Prévenir l'échec", Revue française d'administration publique, no 87, 375-39I, I998, p. 24. 
une adaptation aux conditions qui prévalent dans ces milieux ${ }^{19}$. Après avoir succinctement décrit le cadre théorique dans lequel s'inscrit notre étude, précisons à présent la démarche méthodologique devant servir à vérifier nos hypothèses de recherche.

\section{Méthodologie adoptée}

Afin de comprendre la place qu'occupe la réforme de la fonction publique en République Démocratique du Congo, nous avons jeté notre dévolu sur une démarche méthodologique qualitative.

Dans un premier temps, nous avons mené une étude historique se basant sur les écrits scientifiques et les documents officiels à notre disposition afin de dresser un portrait le plus fidèle possible des mutations engagées depuis les années 1990.

En outre, nous avons complété cette première méthode par la réalisation de plusieurs entretiens directs avec les parties prenantes de ce processus de réforme en RDC, notamment les hauts fonctionnaires, les fonctionnaires de catégories moyenne et inférieure $^{20}$, les experts chargés de concevoir et d'impulser la réforme $e^{21}$ et deux acteurs de la coopération technique belge dont l'un était coordonnateur du projet de réforme de l'administration publique en République Démocratique du Congo avant que ce projet touche à sa fin en décembre 2008.

Nous avons ainsi réalisé 35 entretiens individuels dont 30 avec les hommes et 5 avec les femmes. L'objectif poursuivi par la réalisation de ces entrevues est d'obtenir des informations sur l'opinion que se font ces acteurs par rapport à la réforme engagée au sein de la fonction publique congolaise. L'entretien permet donc de cerner la signification que les interviewés donnent aux mutations administratives en cours. Il nous permet également de savoir si ces changements sont bien vécus ou font l'objet d'une résistance ou si ceux-ci sont désapprouvés.

Par ailleurs, les informations recueillies lors des entretiens ont été retranscrites et analysées de manière à respecter les critères de scientificité propres à la méthode qualitative. Car, celle-ci «tend avant tout à vouloir comprendre le pourquoi et le comment des événements dans des situations concrètes ${ }^{22}$."

Il convient de noter que la réforme de la fonction publique est un processus historique en soi. Ce contexte historique propre à chaque pays permet de mieux comprendre l'évolution du droit, en l'occurrence du droit constitutionnel et du droit administratif. Il

\section{J. I. Gow, op. cit., p. 56.}

Il s'agit des fonctionnaires revêtus des grades de Secrétaire général, du directeur, du chef de division, du chef de bureau, les attachés du bureau, les agents de bureau et les huissiers. Voire l'article 18 de la Loi n ${ }^{\circ} 8 \mathrm{I}$-003 portant statut du personnel de carrière des services publics de l’État. (J.O.Z., noI5, Ier août I98I, p. II) et OrdonnanceLoi n 82-oII du I9 mars I982, art. Ier. - Les emplois auxquels sont affectés les agents soumis au présent statut se divisent en emplois de commandement, de collaboration et d'exécution.

Les experts dont question sont une dizaine de fonctionnaires congolais qui ont intégré la structure dénommée Groupe Projet Fonction Publique, structure chargée de concevoir et d'impulser la réforme administrative en RDC, à la suite d'une sélection rigoureuse organisée par la Belgique au travers son Service Public Fédéral Personnel et Organisation, l'équivalent du Ministère de la fonction publique et la Coopération Technique Belge.

F. Wacheux, Méthodes qualitatives et recherche en gestion, Ed Economica Gestion, I996. 
offre aussi la possibilité d'avoir une meilleure appréciation de l'environnement politique du pays examiné. Par conséquent, cette chronologie relate les évènements importants de I990 à 2014 en lien direct ou indirect avec notre objet de recherche.

\section{Facteurs et enjeux de la réforme de la fonction publique en Afrique Subsaharienne}

Il ne serait pas superfétatoire de rappeler que la réforme de la fonction publique observée ces trois dernières décennies dans le continent africain et en Afrique subsaharienne en particulier tire ses origines de deux facteurs que sont les programmes d'ajustement structurel d'une part et les réclamations incessantes de changement faites par la société civile de certains États africains d'autre part.

En effet, ces revendications avaient abouti vers les années I99o à l'organisation soit de Conférences nationales, soit des États généraux de la fonction publique. La réforme du secteur public a longtemps été au centre des questions de réforme socio-économique et politique sur le continent.

Au début des années I980 quand les programmes d'ajustement structurel commencèrent à s'imposer, le secteur public, aspect le plus visible de l'État interventionniste, était constamment attaqué par le Fonds Monétaire International (FMI) et la Banque mondiale (BM). La fonction publique était, en effet, considérée comme démesurée, inefficace, et disproportionnée, siège de toutes sortes de relations «néo-patrimoniales" et "clientélistes » et source de déficits, ce qui favorisait l'inflation.

Pour ces raisons, il fut inconcevable que les économies des pays africains puissent être structurellement ajustées sans une rationalisation radicale du secteur public, de son rôle, de son mode de fonctionnement et de son mandat. Cette approche de la réforme du secteur public encouragée par les institutions de Bretton Woods est un mélange de commercialisation, de privatisation et de liquidation, mis en œuvre en différentes phases.

Une attention particulière fut alors portée à la réforme de la fonction publique dans la plupart des pays africains, cet exercice prenant la forme de réduction à grande échelle du nombre des travailleurs dans ce qui fut initialement appelé ajustement ${ }^{23}$.

Si la réforme de la fonction publique en Afrique subsaharienne tire ses origines des recommandations faites aux États africains par les Institutions de Bretton Woods, l'autre raison qui la justifie est qu'elle a été, dans certains pays, le fruit d'un consensus.

La réforme de la fonction publique béninoise affirmait Célestin Monteiro, enseignant à la Faculté de Droit et Sciences Politiques de l'Université d'Abomey-Calavi (Bénin), trouve son fondement dans les résolutions de la Conférence des forces vives de la nation et des États généraux de la fonction publique et de la modernisation administrative ${ }^{24}$. Ces deux instances ont affirmé la nécessité de mener des actions hardies susceptibles

23 Codesria, Réformes du secteur public en Afrique : rétrospectives et perspectives, Conférences commémorative Guy MHONE sur le Développement, 2009, p. 2.

24 Adjinako, quotidien d'informations générales et d'annonces béninois, "Réforme administrative au Bénin: l'Alliance ABT dénonce l'initiative du Gouvernement", paru, le 22 avril 2013, http//newes.acotonou.com/ h/3236html, consulté le I6 août 2013 . 
d'élever le niveau de vie des populations et de conduire le pays à un réel développement socio-économique. Il devra en être ainsi parce que l'État républicain choisi comme forme d'organisation politique depuis les indépendances en I96o ne saurait demeurer statique, car tout bouge autour de lui ${ }^{25}$.

C'est dans cette optique qu'il convient d'inscrire la tenue du I2 au i6 décembre I994 des États généraux de la fonction publique et de la modernisation de l'administration béninoise. Ces assises avaient assigné à la réforme administrative le passage d'une administration de commandement à une administration de développement.

Pour y parvenir, quelques recommandations avaient été faites, notamment la clarification des missions et des objectifs de l'État; la réorientation de la culture administrative vers le mérite, le service public et le contrôle de gestion et de résultats; l'utilisation dans l'administration des techniques du management privée ${ }^{26}$.

Toutefois, il convient de souligner que depuis les états généraux de la fonction publique et de la modernisation de l'administration de 1994, des tentatives de réformes menées ont été peu concluantes, comme l'affirme le ministre béninois du Travail et de la Fonction publique, Martial Souton ${ }^{27}$.

«L'heure est venue pour le gouvernement béninois d'adopter une stratégie globale de réforme de la fonction publique en vue de rendre celle-ci plus performante et apte à répondre aux nombreux défis qui s'imposent au développement du pays, a-t-il déclaré ${ }^{28}$."

"La mise en cuvre effective de cette stratégie permettra de régler les nombreux problèmes qui se posent à la fonction publique béninoise à travers trois orientations stratégiques no-tamment l'amélioration du cadre organisationnel et juridique de la fonction publique; l'amé-lioration de la gouvernance de la fonction publique et la modernisation du système de ges-tion des ressources humaines de l'Etat ${ }^{29}$ ", a-t-il expliqué.

Mais pour bon nombre d'observateurs de ce processus de modernisation de l'administration béninoise le changement tant souhaité par les autorités politiques tarde à venir.

En effet, d'aucuns estiment que les efforts de réforme déployés par le gouvernement depuis plus d'une décennie sont très peu perceptibles tant au niveau de la majorité des agents de l'Etat que des usagers de l'administration.

Car, la fonction publique béninoise est toujours gangrenée par plusieurs sortes des dysfonc-tionnements, dont les plus visibles sont: «lenteur dans la mise en œuvre des actions de ré-formes; non application effective de la gestion axée sur les résultats; absence d'une véritable planification stratégique des ressources humaines de l'Etat et enfin

O. Batoko, «La réforme administrative au Bénin: genèse et déploiement d'une politique publique», 2003, $<$ http//democratie.francophonie.org/IMG/pdf/reforme_admin.Bénin, p. I07, consulté le I6 août 2013.

O. Batoko, op. cit., p. I08-109.

Afriquinfos, Le Bénin adopte une stratégie globale de réforme de la fonction publique, le 22 avril 2015, http ://www.afriquinfos.com/articles/20I4/4/23/benin-adopte-strategie-globale-reforme-fonctionpublique-25I77I.asp, consulté le I5 juin 2015.

Afriquinfos, op cit.

Idem. 
l'inefficacité dans la ges-tion des procédures relatives à la carrière des agents de l'Etat ${ }^{30}$." Enfin, l'Assemblée nationale de la République du bénin a adopté en séance du 02 avril 2015 la Loi n²015-I8 portant statut général de la fonction publique. L'un des axes de la réforme en cours au Bénin, cet instrument était attendu depuis près de dix ans et vient abroger la loi n 86-or3 du 26 février 1986 qui régissait jusque-là des agents permanents de l'Etat ${ }^{3 \mathrm{I}}$.

Elle se donne la noble ambition de rendre la fonction publique béninoise performante. Aussi, fixe-t-elle le régime juridique applicable à l'ensemble des agents de la fonction publique ainsi que les principes fondamentaux de gestion des emplois publics.

Selon le rapport de la commission des lois qui s'est penché sur le dossier avant de le sou-mettre à l'examen en plénière, le projet de loi portant statut général de la Fonction publique au Bénin, comporte beaucoup d'innovations ${ }^{32}$. Les plus importantes concernent les condi-tions générales de recrutement dans la fonction publique qui sont désormais fixées comme suit: 35 ans au plus pour les agents des catégories $\mathrm{C}$ et $\mathrm{D}, 40$ ans au plus pour les agents des catégories $\mathrm{B}$ et $\mathrm{A}^{33}$. L'autre innovation de ce texte porte sur l'âge d'admission à la retraite des fonctionnaires. Celui-ci est fixé à 65 ans d'âge pour les professeurs titulaires et les maîtres de conférences ; 63 ans d'âge pour les maîtres-assistants; 62 ans d'âge pour les as-sistants des universités nationales du Bénin [...] ${ }^{34}$.

Nous osons croire qu'avec l'avènement de ce nouveau statut des agents de l'Etat, le Bénin pourrait continuer sans relâche sa marche vers la matérialisation des autres chantiers de sa réforme, en vue de la mise en place d'une fonction publique performante au bénéfice de ses citoyens.

Dans un tout autre registre, la demande pour une participation plus effective des populations aux affaires publiques a justifié les «consultations populaires » initiées par le président Mobutu en I99o en vue de recueillir l'opinion que les citoyens se faisaient sur le système de parti unique en place depuis I $_{9} 65$.

De même, la pression populaire a rendu possible la tenue entre I99I et I992 d'une «Conférence Nationale Souveraine» (CNS) dont l'objectif était de faire le point sur l'état de la gouvernance dans le pays depuis son indépendance en I960, et de jeter les bases d'un système nouveau de gouvernance débarrassé de la dictature du parti unique ${ }^{35}$.

24 heures au Bénin, L'intégralité de la zème Conférence publique de l'Alliance ABT sur le Thème «La réforme de la fonction publique béninoise en question", 22 avril 2013, http://24haubenin.info/?L-integralite-de-la-3eme, consulté le I5 août 2015 .

3I Fraternité, Vote de la loi portant statut général de la fonction publique: Vers une administration publique performante, le 03 avril 2015, http://fraternitebj.info/politique/article/vote-de-la-loi-portant-statut, consulté le I8 novembre 2015 .

32 La Tribune de la capitale, Administration publique au Bénin: Voici les nouvelles conditions de recrutement et d'admission à la retraite, le o6 avril, 2015, atribunedelacapitale.com/actualite/1537-administration-publiqueau-benin-voici-les-nouvelles-conditions-de-recrutemen, consulté le i9 novembre 2015.

33 République du Bénin, article 12 point 5 de la Loi n 2015-I8 portant statut général de la fonction publique.

34 République du Bénin, article 263 de la Loi n 2015-I8 portant statut général de la fonction publique.

35 Phambu Ngoma-Binda, J. Otemikongo Mandefu Yahisule et al., Démocratie et participation à la vie politique: une évaluation des premiers pas dans la IIIème République, étude d'AfriMap et de l'Open Society Initiative for Southern Africa, novembre 2010, p. 3. 
Cependant, il faut souligner que la réforme de la fonction publique a été pour quelques États du continent noir une initiative gouvernementale.

En novembre 2003, le gouvernement de la République Démocratique du Congo a lancé la troisième réforme administrative d'envergure nationale après l'indépendance. Cette réforme, qui a démarré au sein des administrations centrales, était prévue pour se poursuivre dans les mois et les années à venir auprès des services décentralisés ou déconcentrés en province. "Pour ce faire, le gouvernement comptait sur trois facteurs : l'accord unanime des acteurs internes et des partenaires extérieurs sur la nécessité de réformes structurelles, l'appui de la communauté internationale et l'implication massive des fonctionnaires et agents de l'État ${ }^{36}$."

\section{Descriptif de la réforme de la fonction publique Congolaise}

Fin 2003, le président Joseph Kabila signait le décret nº3/035 du I3 novembre 2003 portant création, organisation et fonctionnement de la Commission interministérielle de pilotage de la réforme de l'administration publique (CIPRAP), consacrant ainsi le démarrage effectif de la mise en œuvre de la réforme ${ }^{37}$.

Selon le décret susmentionné, la CIPRAP a pour mission de diriger et d'orienter la réforme de l'administration publique, de décider des objectifs, du plan d'action et du budget, d'assurer la préparation et le contrôle des actions, d'évaluer les résultats et de rechercher le financement nécessaire ${ }^{38}$.

Cette structure est présidée par le ministre de la fonction publique et se réunit, sur convocation de son président, au moins une fois par trimestre. Pour lui garantir un bon fonctionnement, la CIPRAP dispose d'un Comité technique doté d'un secrétariat permanent qui assure la gestion quotidienne et collabore avec les institutions, ministères et organismes concernés par la réforme de l'administration publique.

Appelée à transformer aussi bien les organismes de l'administration publique que le personnel de ceux-ci, la réforme a été dotée d'un double objectif: la mise sur pied d'une administration publique performante capable d'assumer les missions essentielles de l'État et l'émergence d'un nouveau type d'agent efficace, motivé et revalorisé39.

Les autorités avaient donné la part belle aux partenaires au développement dans la conduite de la réforme pour deux raisons : premièrement, l'implication de ces derniers crédibilisaient le processus et permettaient également le transfert de savoirs et de compétences. Deuxièmement, en faisant intervenir massivement les acteurs internationaux, le gouvernement congolais visait surtout leurs financements. En effet, la réforme en cours en République Démocratique du Congo n'a jamais fait l'objet d'une appropriation financière nationale. La politique de la main tendue en direction des partenaires bilatéraux ou multilatéraux semble être «la stratégie de financement» de ladite réforme.

\footnotetext{
36 R. Diumasumbu, «Réforme administrative de la République Démocratique du Congo: Leçons et travers du passé», Afrique contemporaine, 2008/3 n²27, p.95, p. 95-I06. DOI: 10.3917/afco.227.0095.

37 R. Diumasumbu, op.cit., p. Ioo.

38 R. Diumasumbu, op.cit., p. Ioo.

39 Idem, p. Ioo.
} 
En outre, les réformateurs ont voulu que la réforme soit intégrale et qu'elle s'attaque de manière globale aux maux dont souffre la fonction publique congolaise.

A cet égard, la réforme devrait être une réforme intégrée car tenant compte des différents acteurs et leurs intérêts respectifs; une réforme novatrice et progressive qui se voulait porteuse de nouvelles méthodes de gestion et de fonctionnement de l'appareil administratif en vue de la bonne gouvernance.

Enfin, une réforme qui devait commencer par les administrations centrales avant de s'étendre en provinces ainsi qu'aux entités décentralisées ou déconcentrées, tel que dispose l'article 3 de la Constitution congolaise.

Le cadre stratégique de la gouvernance administrative défini par le PNUD en 2007 portait sur la rationalisation du cadre institutionnel et organisationnel, la modernisation de la gestion des ressources humaines et leur valorisation avec un accent particulier sur la place des femmes dans la fonction publique, la promotion de l'approche qualité et de l'emploi dans le secteur public.

L'intervention dans ces différents axes devait être précédée par trois actions prioritaires (recensement des fonctionnaires et agents de l'État, mise à la retraite des agents atteints par la limite d'âge, et audit et renforcement des systèmes de la paie des fonctionnaires) visant la maîtrise des effectifs et de la masse salariale ${ }^{40}$.

Il ressort de la lecture de fiche projet appui à la réforme des administrations publiques du programme gouvernance du PNUD en République Démocratique du Congo que l'objectif global poursuivi était de mettre sur pied une administration saine, moderne et performante capable d'assumer les missions de l'État et de contribuer au développement du pays; de susciter l'émergence d'un nouveau type d'agent de l'État, bien formé, efficace, revalorisé; de rendre cette administration capable d'offrir un service public de qualité; promouvoir la bonne gouvernance et l'éthique au sein des services de l'État ${ }^{4 \mathrm{I}}$.

\section{Bilan de la réforme de la fonction publique en République Démocratique du Congo : essai d'une évaluation}

En ce qui concerne le bilan de la réforme de la fonction publique en RDC, disons tout de suite que celui-ci se base sur des observations, des échanges et entretiens réalisés entre janvier 2007 et octobre 2014 avec les différentes parties prenantes à ce processus, à savoir : les usagers des services publics, les fonctionnaires, les experts des structures de réforme, les experts de la coopération technique belge et quelques secrétaires généraux des administrations.

Toutefois, à la lumière de ce qui a été fait jusqu'à ce jour, force est de constater que peu d'actions inscrites dans les différents cadres stratégiques et autres documents relatifs au présent processus de réforme de la fonction publique en RDC ont été menées à bien. 
Globalement les objectifs spécifiques qui apparaissent en filigrane dans la Note conceptuelle sur la stratégie révisée de la réforme de l'administration publique congolaise n'ont pas été atteints ${ }^{42}$.

Il faut souligner ici que le succès d'une réforme, selon la littérature empiriste, compte sur des facteurs de conscience de besoin, d'investissement, d'engagement et d'ancrage, de communication, d'animation et d'apprentissage ${ }^{43}$.

«Or, tout bien considéré, on a comme l'impression que les cercles du pouvoir politique et administratif ne partagent pas la même perception de la réforme entreprise, car ceux-ci sont les premiers responsables du niveau et de la qualité de l'investissement consenti : il leur faut accepter clairement le projet, garantir les fonds, assurer la coordination, assumer les conflits, etc. ${ }^{44}$ »

La nécessité d'entreprendre une réforme doit être largement ressentie par tous les stakeholders au processus, faute de quoi, les dossiers de réforme risqueraient de ne pas se trouver en haut de l'agenda des priorités du gouvernement lorsque d'autres besoins se manifesteront à lui ${ }^{45}$.

En sus, l'on a assisté à de batailles presque rangées entre bailleurs dans l'approche méthodologique de conduite de la réforme de l'administration publique en RDC. Cette situation est, à notre avis, l'un des facteurs d'insuccès que l'on pourrait mettre dans le passif de ce processus de changement, d'autant plus qu'il y a eu une fracture dans la perception de cette entreprise entre d'une part les experts nationaux partisans de l'approche belge et d'autre part, ceux défenseurs de la méthodologie prônée par le PNUD.

Bien trop souvent on a cru, comme le soutient Jacques Bourgault, que «de bonnes idées, des besoins bien ressentis et documentés suffisent à garantir le succès de la réforme. Une réforme ne peut faire l'économie de s'appuyer sur de solides fondements techniques en amont et sur un appui sophistiqué en aval. Elle doit compter sur des investissements qui prennent diverses formes, en l'occurrence: les moyens humains, financiers et matériels, recherche, énergie politique, acceptation des conflits inhérents aux changements ${ }^{46}$.»

A ce propos, il faut noter que l'investissement consenti par le gouvernement est quasi insignifiant, car le soutien financier que connait la réforme vient de partenaires bilatéraux ou multilatéraux. A ce sujet, l'étude de Raphaël Diumasumbu Mukanga est révélatrice. Démocratique du Congo de septembre 2012, p. I2.

43 P. Weller, «Reforming the Public Service: What has been Achieved and how can it be Evaluated?» in Weller P., Forsters J. et Glyn D., 222-236.

44 J. Bourgault, «Conditions de succès d'implantation des réformes administratives en Afrique: leçons apprises au sujet des stratégies d'appui au changement", Mondes en développement, 2006/I no I33, p. 63-82. DOI: I0.39I7/ med.133.0063, pp. 65 et 66.

45 Idem, p. 65.

46 Ibidem, p.66. 
Plusieurs partenaires assistent en effet le gouvernement dans le financement de la réforme de son appareil administratif. Outre la rationalisation des structures, emplois et effectifs, la mise à disposition d'une contribution à la poursuite du recensement en province, le plan de communication et quelques activités ad hoc, la Belgique a financé le fonctionnement du Comité technique de la Commission interministérielle de pilotage de la réforme de l'administration publique, celui des Comités de stratégie, du Groupe projet fonction publique, des Cellules de réforme des ministères pilotes et des Cellules thématiques de réforme ${ }^{47}$.

Il convient de noter que la Belgique a conclu, en mai 2003, une convention spécifique mettant en place un fonds d'appui à la réforme de l'administration publique de la RDC doté d'une enveloppe initiale de 4 millions d'euros pour financer des actions de recensement du personnel, des études sur le statut administratif et salarial, l'élaboration détaillée des cadres organiques des différents ministères, les plans de formation ainsi que des appuis aux structures de pilotage ${ }^{48}$.

La République sud-africaine, de son côté, a participé à hauteur de 7 millions de dollars US au financement du recensement général des agents et fonctionnaires de l'État ainsi qu'à la mise en place d'une école nationale d'administration.

La Banque mondiale a financé la mise à la retraite des agents excédentaires, à hauteur de 45 millions de dollars US et la France, l'élaboration de la procédure transitoire simplifiée de paie des agents et fonctionnaires de l'État.

Par ailleurs, «le Projet de réforme et de rajeunissement de l'administration publique, dont l'accord de financement a été signé en février 2014 entre le gouvernement de la RDC et la Banque mondiale pour un montant de 77 millions de dollars américains s'inscrit dans la volonté commune d'utiliser désormais le système national pour la mise en œuvre des projets financés par la Banque mondiale de contribuer ainsi au renforcement des capacités des institutions publiques nationales ${ }^{49}$."

Le PNUD, quant à lui, a financé la rédaction des statuts juridiques et régimes de protection sociale, les études sur la création de l'école nationale d'administration, le dialogue social, la rationalisation des structures, emplois et effectifs ainsi que l'élaboration d'un plan de communication de la réforme ${ }^{50}$.

De ce qui précède, il est aisé de comprendre qu'on ne peut réussir une réforme sans assurer de manière continue les moyens matériels nécessaires aux chantiers retenus. Enfin, disons un mot sur l'ancrage de la réforme de la fonction publique congolaise.

«Entendu comme le point de son rattachement au système décisionnel national et au système du passage à l'acte, son efficacité fait en sorte qu'il devient impossible, pour les forces du statu quo, de la freiner, de l'atténuer et ou de la détourner. Il se fait autour

\footnotetext{
47 R. Diumasumbu, op.cit., p. I03.

48 Idem, p. I03.

49 E. Pinto Moreira, Discours de Monsieur Emmanuel Pinto Moreira, Représentant résidant ai de la Banque mondiale pour la République Démocratique du Congo, prononcé au Forum International sur la Réforme de l'Administration Publique en République Démocratique du Congo, le o2 octobre 2014. 
d'acteurs et d'un événement particulièrement significatif (budget, agenda de réconciliation nationale, etc.). Il implique à la fois des acteurs politiques et administratifs ${ }^{5 \mathrm{I}}$ ".

Nous constatons, néanmoins, sur le terrain non seulement le manque d'une vision partagée mais également le déficit criant de coordination. En effet, le Ministère de la fonction publique ne semble pas avoir la carrure nécessaire pour imprimer le changement tant attendu au sein de l'administration publique de la RDC. A titre d'illustration, en sa qualité du Président de la CIPRAP, le ministre de la fonction publique n'a pas su organiser en ro ans plus de 2 réunions de cette structure, alors que le texte la créant dispose que la CIPRAP que préside le ministre de la fonction publique se réunit, sur convocation de ce dernier, au moins une fois par trimestre.

L'une des raisons qui pourrait justifier cette situation, c'est notamment le fait que le ministre de la fonction publique dans le contexte institutionnel congolais n'est pas préséant par rapport à ses collègues du budget ou des finances par exemple. Dans la pratique politique congolaise, celui-là n'a pas le même prestige que ceux-ci.

En raison de son caractère transversal et du principe selon lequel une réforme devrait s'adapter à son contexte à la fois historique, institutionnel, voire culturel, nous estimons, pour notre part, que son encrage devrait être soit à la présidence de la République ou tout le moins à la primature.

Il convient, toutefois, de souligner que le retard observé dans la mise en place du cadre juridique de la réforme de la fonction publique est de nature à freiner la mise en place de la fonction publique provinciale et le transfert des compétences aux provinces, comme le postule la décentralisation que préconise la Constitution de la RDC en son article 3 .

En dépit du catalogue des bonnes intentions que constituent les différents documents élaborés dans le cadre de la présente réforme de l'administration publique congolaise, il est un secret de Polichinelle que d'affirmer que ce processus en cours au CongoKinshasa est un échec aux yeux des citoyens.

Pour s'en convaincre, il suffit de mesurer la portée de cette phrase de Théodore Trefon lorsqu'il affirme qu'une récente enquête sur la façon dont les congolais perçoivent l'État comprenait la question suivante : «Si l'État était une personne, que lui feriez-vous?» «Je le tuerais" telle était la réaction sans équivoque de la plupart des répondants ${ }^{52}$.

Mais il faut, cependant, relever que les fonctionnaires apprécient particulièrement le fait que la présente réforme soit la première qui leur accorde une place importante dans sa mise en œuvre. Ils se mobilisent donc pour mériter la confiance qui leur est témoignée par le pouvoir exécutif.

«La grande interrogation est de savoir combien de temps va durer cet état de grâce car, la réforme n'ayant pas encore incité aux indispensables assainissements des pratiques, on doit se demander ce qu'il en adviendra quand ce travail d'assainissement dans

$5 \mathrm{I} \quad$ J. Bourgault, op. cit, p. 68.

52 T. Trefon, «Les obstacles administratifs à la réforme en République démocratique du Congo », Revue Internationale des Sciences Administratives, 2010/4 Vol. 76, p. 735-755. DOI : I0.3917/risa.764.0735, p. 746. 
la gestion du personnel démarrera pour de bon et que les organisations syndicales entreront en piste 53 ".

Au demeurant, en analysant de près les objectifs généraux et spécifiques visés par la présente réforme, l'on est en droit d'affirmer que ceux-ci n'ont pas été atteints à leur grande majorité.

Pour l'illustrer, nous citons comme exemple l'axe de réforme relative à la révision du cadre juridique de la fonction publique congolaise. En effet ce volet de la réforme demeure une préoccupation jusqu'à ce jour. Comment comprendre que les travaux sur le nouveau statut des agents et personnel de l'État, le texte devant régir la fonction publique n'a pas fait l'objet de délibération en conseil des ministres jusqu'en début 2013, alors que le projet de texte avait déjà été élaboré par les experts en 2008 et avait d'ores et déjà reçu le quitus du ministre de la fonction publique de l'époque. Cette priorité ne demande quasiment pas des moyens financiers, seule la volonté politique peut permettre la matérialisation de ce chantier de réforme.

De même, la fixation des cadres et structures organiques des services centraux des ministères du gouvernement et des administrations de certaines institutions politiques telles que les chambres basse et haute du Parlement, se fait toujours attendre.

A l'heure actuelle, on peut se permettre d'affirmer que la réforme en cours est à deux vitesses : les Ministères tels que : environnement, plan, budget, finances, agriculture, justice, santé, etc., pour ne citer que ceux-ci, ont vu leur cadre organique validé par le Ministère de la fonction publique pour la simple et bonne raison que leurs administrations ont bénéficié de financements des partenaires bilatéraux ou multilatéraux.

En revanche, ceux des Ministères n'ayant pas trouvé "grâce" auprès de ces derniers sont abandonnés à leur triste sort. C'est notamment les cas : communication et médias, culture et arts, défense nationale et anciens combattants, développement rural, genre et famille, jeunesse, sports et loisirs...

De toutes les façons, le Ministère de la fonction publique qui est artisan de ce travail de rationalisation des missions, structures, emplois et effectifs au sein de l'administration publique demeure impuissant et ne sachant quoi faire, parce que ne disposant pas de moyens financiers et matériels nécessaires pour mener à bien sa politique.

Lors de son intervention en mars 2013, le ministre de la fonction publique a relevé que l'administration publique congolaise est confrontée aux multiples problèmes en rapport avec son organisation et son fonctionnement notamment les conditions matérielles et de travail des agents et fonctionnaires, le déficit en équipement des Nouvelles Technologies de l'Information et de Communication, les règles d'éthique et de déontologie ainsi que le vieillissement et l'insuffisance des capacités de la majeure partie du personnel ${ }^{54}$.

Loin d'être un constat lamentable du tableau qu'affiche son secteur, le ministre de la fonction publique fait, par la même occasion, probablement consciemment ou inconsciemment un aveu d'incapacité pour la réalisation des objectifs spécifiques qu'il a pour-

54 Digital Congo, "Le ministre KIBALA motivé par le souci de mettre de l'ordre dans son secteur", édité le I6/03/2013, http://www.digital.congo.net/article/90474. 
tant identifiés et qui sont inscrits dans sa Note conceptuelle sur la stratégie révisée de la réforme de l'administration publique de la République Démocratique du Congo de septembre 2012.

Aux dernières nouvelles, nous avons appris que l'actuel locataire du bâtiment administratif du Ministère de la fonction publique a décidé, non pas par arrêté ministériel, mais par personne interposée, précisément le Secrétaire général de la fonction publique en charge du personnel actif, de mettre fin aux structures de réforme dont l'une instituée par le Décret du chef de l'État, pour des raisons inavouées.

On aurait appris que sentant venir le financement promis par la Banque mondiale, cette autorité voudrait, même dans l'hypothèse plausible de son départ de ce département, laisser l'un de ses proches, coordonnateur d'une de structures qu'il aurait créées pour le besoin de la cause, en vue de substituer celles qui ont fait leur preuve voilà déjà une décennie.

Face à ces agissements irresponsables qui n'honorent pas son auteur, l'on est en droit d'être sceptique et légitimement se demander si le changement promis en 2003 est encore possible dans la fonction publique congolaise.

Il convient de rappeler, à ce stade, que la réforme ne fait pas bon ménage avec l'incompétence des acteurs appelés à l'impulser, l'absence ou la faiblesse de leadership, le tribalisme, le clientélisme, bref des antivaleurs de toutes sortes.

Dans les circonstances susmentionnées, l'on risque de donner raison aux nombreux auteurs qui ont souligné les effets pervers rencontrés au niveau de la réforme de certaines fonctions publiques africaines dont celle de la RDC.

Ainsi, Achille Mbembe écrit: «En l'absence d'autres débouchés, la lutte de chaque famille est orientée vers l'objectif de placer l'un des siens à un poste dans la fonction publique, puisqu'il y va de sa survie [...]. Des filières très complexes sont ainsi inventées dans le but d'augmenter les fortunes et de centraliser les «dépouilles» vers le haut, tout en fermant l'accès à la "table» aux autres prétendants ${ }^{55}$."

«Si beaucoup de politiques de réforme sont pertinentes au niveau théorique, leur bonne mise en œuvre ne s'est cependant pas matérialisée, en grande partie en raison de l'absence de structure administrative fiable et motivée. Il faut trouver un juste milieu entre la dimension utopique du souhaitable et celle, pragmatique, du réalisable ${ }^{56}$ "

Dans la quête de cet équilibre entre la situation souhaitée et celle dans laquelle se trouve réellement la réforme de la fonction publique en République Démocratique du Congo, que le Premier Ministre, Augustin Matata Ponyo, a procédé le jeudi 2 octobre 20I4, à l'ouverture officielle du Forum international de haut niveau sur la réforme et modernisation de l'administration publique, devant des députés nationaux, des membres

\footnotetext{
55 M. Rambourg, Aspects méthodologiques de la réforme des fonctions publiques africaines le: cas des pays francophones au sud du Sahara, Réunion sur la réforme administrative en Afrique francophone, ONU, New York, 4 juin 1997 , p.15.

56 T. Trefon, op. cit., p. 749.
} 
de son gouvernement, des gouverneurs de provinces, des représentants des partenaires de la RDC et autres invités conviés à cette réunion, qui avait pour principal thème : «Rôle de l'administration publique pour la transformation d'Etat post-conflit en pays émergeant: Quelles réformes en vue de moderniser l'Administration publique pour l'efficacité et l'accessibilité des services publics en RDC».

$\mathrm{Au}$ risque de considérer a priori cette rencontre comme un énième rendez-vous manqué, nous attirons, néanmoins, l'attention de nos lecteurs à deux observations ciaprès : premièrement, nous estimons que la terminologie employée pour nommer ce forum est inappropriée et inutilement prétentieuse.

En effet, pourquoi les autorités congolaises parlaient-elles d'un «forum international de haut niveau»? A notre avis, c'est présomptueux de leur part d'avancer des termes tels que: «de haut niveau " pour désigner des assises qui ont pourtant été organisées par elles-mêmes, en vue de rechercher des solutions durables pouvant garantir un succès au processus de réforme et de modernisation de l'appareil de l'État.

En outre, nous sommes, une fois de plus, sceptiques quant à la terminologie usitée, car le profil de certains participants à ce dernier forum sur la réforme de l'administration publique congolaise ne nous semblait pas répondre aux critères universellement admis pour le prétendu « haut niveau », qualificatif collé audit forum.

$\mathrm{Au}$ moment où nous sommes en train de mettre la dernière main à cette étude, la réalité sur le terrain atteste que la réforme de la fonction publique est en train de battre de l'aile, malgré l'arrogance des autorités congolaises. Deuxièmement enfin, les problèmes visés par ledit forum ne sont pas nouveaux. En effet, ceux-ci avaient déjà été abordés lors des autres rencontres précédentes.

Pour rappel, les travaux de ce forum ont tourné autour de trois thèmes centraux ciaprès : contexte, défis et enjeux de la réforme et modernisation de l'administration publique, rationalisation et gestion innovante des ressources humaines de l'administration publique et enfin stratégie pour la transformation de l'administration en une administration publique de développement et la fourniture des services publics de qualité aux usagers.

Comme vous pouvez le constater, les trois axes de problèmes examinés lors de cette réunion d'octobre 2014 ne sont ni moins ni plus les axes prioritaires déjà visés par le cadre stratégique de la réforme de 2003 et revisité en 2007. Pourquoi doit-on revenir sur ce qui déjà faisait l'objet des diagnostics précédents? C'est, une fois de plus, un véritable gaspillage d'argent et d'énergies que de revenir sur des problématiques auxquelles des pistes de solutions avaient d'ores et déjà été données en leur temps.

En revanche, ce dont la RDC a réellement besoin pour faire aboutir la réforme de sa fonction publique et partant de celle de son État, c'est l'implication effective des autorités politiques ainsi que celle des autres couches sociales de la population congolaise; afin qu'ensemble, dans un élan patriotique et inclusif, l'on puisse jeter les bases solides et saines d'une véritable réforme de la fonction publique gage de son développement, au travers une réflexion profonde, dans l'unique but de dégager une méthodologie adéquate de conduite de ce processus, en prenant en compte les contraintes de toutes sortes. 


\section{Conclusion}

«Le problème de la gestion des personnels de l'État a pris, ces dernières années dans les pays en développement et singulièrement dans les pays francophones d'Afrique au sud du Sahara, une acuité particulière à des degrés divers ${ }^{57}$."

L'efficacité de la fonction publique est progressivement apparue comme une condition primordiale à une bonne gouvernance qui garantit une réalisation effective des plans nationaux de développement et des stratégies de réduction de la pauvreté, ce qui au jour d'aujourd'hui n'est pas encore le cas en RDC malgré toutes ces années de réforme de sa fonction publique.

Pour arriver à une fonction publique efficace, la République Démocratique du Congo doit faire face à divers grands défis et tenter de les surmonter : renforcer les capacités d'analyse, de planification, de suivi et d'évaluation, améliorer les infrastructures, rapprocher les services des citoyens, traduire les orientations politiques en engagements financiers concrets, décentraliser la prise de décisions, les ressources et le pouvoir, gérer de façon plus rationnelle les ressources, stimuler la motivation et la productivité des fonctionnaires sans oublier de lutter contre la corruption qui gangrène l'ensemble de ses strates sociales.

Bien entendu, toutes ces recommandations ne serviraient à rien, si en plus, il n'y a pas un réel engagement politique de la part de son gouvernement.

Dans ce contexte, la réforme de la fonction publique reste en dépit des échecs enregistrés, plus que jamais d'actualité car elle est le seul moyen de mettre en place un service public efficace et de qualité qui soit respectueux des principes fondamentaux des droits de l'homme et du citoyen et à même de promouvoir un environnement propice au développement économique et social dont la République Démocratique du Congo a grandement besoin.

\section{Références}

Adjinako, Quotidien d'informations générales et d'annonces béninois, "Réforme administrative au Bénin: l'Alliance ABT dénonce l'initiative du Gouvernement", 2013 <http//newes.acotonou.com/ $\mathrm{h} / 3236 \mathrm{html}$.

Afriquinfos, Le Bénin adopte une stratégie globale de réforme de la fonction publique, le 22 avril 2015 , $<$ http://www.afriquinfos.com/articles/2014/4/23/benin-adopte-strategie-globale-reforme-fonction-publique-251771.asp

Batoko, $\mathrm{O}$. "La réforme administrative au Bénin: genèse et déploiement d'une politique publique", 2003, http//democratie.francophonie.org/IMG/pdf/reforme_admin.Bénin, I07-II4.

Bourgault, J. «Conditions de succès d'implantation des réformes administratives en Afrique: leçons apprises au sujet des stratégies d'appui au changement", Mondes en développement, 2006/I no I33, DOI : $10.3917 /$ med.I33.0063,63-82.

Butani, K. N., «Implementing Administrative Innovations and Reforms», The Indian Journal of Public Administration, 1966, I2, 612-617.

Caiden G., "Administrative reform», RISA, I968. 
Cabatoff, K., «La réforme institutionnelle de la fonction publique», dans J. Bauer et K. Cabatoff, Bilan de la loi 50 : dynamique du changement de la fonction publique québécoise, Actes du premier colloque sur la réforme et le changement en administration publique, Université du Québec à Montréal, r980, 37-59.

Chapel, Y., "Stratégies et tactiques de la modernisation publique dans les pays en développement» (recueil de textes), Bruxelles, IISA, I972, I82.

Chevalier, J. «Prévenir l'échec », Revue française d'administration publique, I998, no 87, 375-39I.

Chevallier, M. et LOSCHAK, D., Science administrative, vol. I, Paris, LGDJ, I979, I75.

Codesria, Réformes du secteur public en Afrique: rétrospectives et perspectives, Conférences commémorative Guy MHONE sur le Développement, 2009, I-3.

Crozier, M. et Friedberg, E., L'acteur et le système, Paris, Le Seuil, I977, pp. 2 I2 à 2 I4.

Ctrap, La réforme de l'administration publique phase I, Ministère de la fonction publique, Kinshasa, 2007, inédit.

Digital Congo (2013), Le Ministre KIBALA motivé par le souci de mettre de l'ordre dans son secteur, édité le I6/03/2013, http://www.digital.congo.net/article/90474

Diumasumbu Mukanga, R., «Réforme administrative de la République Démocratique du Congo : Leçons et travers du passé», Afrique contemporaine, 2008/3 n 227, DOI : I0.3917/afco.227.0095, 95-106.

Fraternité, Vote de la loi portant statut général de la fonction publique: Vers une administration publique performante, le 03 avril 2015, <http ://fraternitebj.info/politique/article/vote-de-la-loi-portant-statut

Giauque, D. et Caron, D.J., «Réformes administratives et gestions des ressources humaines: comparaison de la Suisse et du Canada ", De Boeck Supérieur, Revue internationale de politique comparée, 2004, 2004/2, Vol. II, 225-240.

Gow J.I., "La réforme institutionnelle de la fonction publique de 1983 : contexte, contenu et enjeux", Politique, $\mathrm{n}^{\circ}$ 6, 1984, 5I-I0I.

24 heures au Bénin, L'intégralité de la zème Conférence publique de l'Alliance ABT sur le Thème «La réforme de la fonction publique béninoise en question", 22 avril 2013, < http ://24haubenin.info/ ?Lintegralite-de-la-zeme.

La tribune de la capitale, Administration publique au Bénin: Voici les nouvelles conditions de recrutement et d'admission à la retraite, le 06 avril, 2015, latribunedelacapitale.com/actualite/1537-administration-publique-au-benin-voici-les-nouvelles-conditions-de-recrutement.

Lemieux, V., Les chemins de l'influence, Québec, PUL, r979.

Livian, Y.-F., Organisation. Théories et pratiques, Paris, Dunod, 1998, 322.

Loi $n^{\circ}$ 2015-18 portant statut général de la fonction publique de la République du Bénin.

Loi, $\mathrm{n}^{\circ} 8 \mathrm{I}-003$ portant statut du personnel de carrière des services publics de l'État (RDC) in J.O.Z., n ${ }^{\circ}$ I5, Ier août Ig8r.

Ministère de la fonction publique, Note conceptuelle sur la stratégie révisée de la réforme de l'administration publique de la République Démocratique du Congo de sepembre 2012.

Ngoma-Binda Phambu, Otemikongo Mandefu Yahisule, J. et Moswa Mombo, L., Démocratie et participation à la vie politique: une évaluation des premiers pas dans la IIIème République, étude d'AfriMap et de l'Open Society Initiative for Southern Africa, Johannesburg, 2010, 264.

Pinto Moreira, E., Discours de Monsieur Emmanuel Pinto Moreira, Représentant résidant ai de la Banque mondiale pour la République Démocratique du Congo, prononcé au Forum International sur la Réforme de l'Administration Publique en République Démocratique du Congo, le 02 octobre 2014.

Piotet, F. et Sainsaulieu, R., Méthodes pour une sociologie de l'entreprise, Paris, Presses de la Fondation nationale des sciences politiques, I994, 374 .

PNUD en RDC, Fiche projet Appui à la réforme des administrations publiques, 2008. en RDC, Appui à l'émergence de la bonne gouvernance en RDC: Note d'orientation, Kinshasa, 2007. 
Rambourg, M., Aspects méthodologiques de la réforme des fonctions publiques africaines le: cas des pays francophones au sud du Sahara, Réunion sur la réforme administrative en Afrique francophone, ONU, New York, I997, 60.

Reynaud, E. et Reynaud J.-D, «La régulation conjointe et ses dérèglements», Le Travail humain, vol. 57, $\mathrm{n}^{\circ} \mathrm{I}, \mathrm{I} 1994,227-238$.

Trefon, T., "Les obstacles administratifs à la réforme en République démocratique du Congo", Revue Internationale des Sciences Administratives, 2010/4 Vol. 76, DOI : 10.39I7/risa.764.0735, 735-755.

Wacheux, F., Méthodes qualitatives et recherche en gestion, Ed Economica Gestion, I996.

Weller, P., «Reforming the Public Service: What has been achieved and how can it be evaluated?» in Weller P., Forster J. et Glyn D., 1993, 222-236. 